\title{
Cystic fibrosis carrier detection using a linked gene probe
}

\author{
MARTIN FARRALL, P.ETER SCAMBLER, KATHERINE WOOD KLINGER*, \\ KEVIN DAVIES, CATE WORRALL, ROBERT WILLIAMSON, AND \\ BRANDON WAINWRIGHT \\ From the Department of Biochemistry, St Mary's Hospital Medical School, University of London, London \\ W2 1PG; and *Integrated Genetics Inc, Framingham, Massachusetts 02139, USA.
}

SUMmARY Cloned DNA markers which are closely linked to the gene defect causing cystic fibrosis have recently been described. These markers are sufficiently informative for carrier detection in $80 \%$ of families where there is a living cystic fibrosis child and unaffected sibs. The tightly linked DNA marker pJ3.11 was used in this study to identify carriers in six families and exclude carrier status in two subjects. Risk calculations for recessive diseases using linked DNA probes may be complex, but useful information for counselling can be obtained in this way.

Cystic fibrosis is one of the most common genetic diseases in North European populations, with an incidence at birth of approximately one in 1800 and a carrier frequency of approximately one in 20 . The biochemical pathology of the disease is uncertain, although recent work has implicated anion transport across membranes as the physiological basis of the mutation. ${ }^{1}$

There are several published methods that claim to detect carriers for cystic fibrosis in family studies or as a population screening test. Qureshi and Punnett ${ }^{2}$ have recently evaluated four methods that rely on detecting cystic fibrosis antigen, lectins, lectin-like substances, or reduced levels of certain proteases in plasma, respectively. The authors conclude that these tests separately or in combination are unreliable for carrier determination in population screening; their usefulness in detecting carriers among unaffected sibs in cystic fibrosis family studies could not be determined. A recent report that bromide excretion is delayed in cystic fibrosis patients and heterozygotes has not yet been independently confirmed. ${ }^{3}$

Genetic linkage between the cystic fibrosis locus and five DNA probes which recognise restriction fragment length polymorphisms (RFLPs) has recently been reported. ${ }^{4-8}$ Two of the probes $\left(\mathrm{pJ} 3 \cdot 11^{4}\right.$ and $\mathrm{met}^{6}$ ) are tightly linked to cystic fibrosis and localise the genetic defect to chromosome $7 q 2 \cdot 1-2 \cdot 2$. The availability of these closely linked

Received for publication 24 March 1986 Accepted for publication 10 April 1986. probes will allow determination of carrier status or prenatal diagnosis using chorionic villus sampling in many informative families. This report illustrates the application of the linked probe $\mathrm{pJ} 3.11$ to carrier detection or exclusion, and shows some of the complexities associated with making predictions for recessive disorders using linked markers.

\section{Materials and methods}

\section{FAMILIES}

The families described here were collected for linkage studies and were ascertained for multiple children affected by cystic fibrosis. No evidence for non-paternity was found using approximately 40 protein, red blood cell antigen, and DNA typings.

\section{DNA HYBRIDISATION ANALYSIS}

DNA was prepared from venous blood samples by standard methods ${ }^{9}$ and $3 \mu \mathrm{g}$ DNA was digested to completion with the restriction endonuclease MspI. The resulting fragments were separated by electrophoresis on a $\mathbf{0 \cdot 8 \%}$ agarose gel and transferred to a nylon membrane (Hybond ${ }^{\mathrm{TM}}-\mathrm{N}$, Amersham) by Southern blotting. ${ }^{10}$

The Southern blot was hybridised overnight at $65^{\circ} \mathrm{C}$ in hybridisation fluid ${ }^{11}$ containing $2.5 \mathrm{ng} / \mathrm{ml}$ of radiolabelled pJ3.11 probe insert, $10 \%$ dextran sulphate, and $500 \mu \mathrm{g} / \mathrm{ml}$ sodium heparin sulphate. The human DNA insert of pJ3.11, which is flanked by HindIII and EcoRI restriction endonuclease sites, was excised from a $1.5 \%$ low gelling temperature agarose gel after the appropriate double restric- 

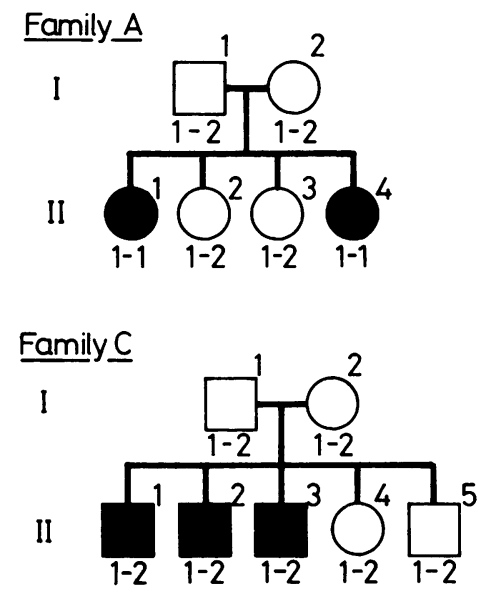

Family E

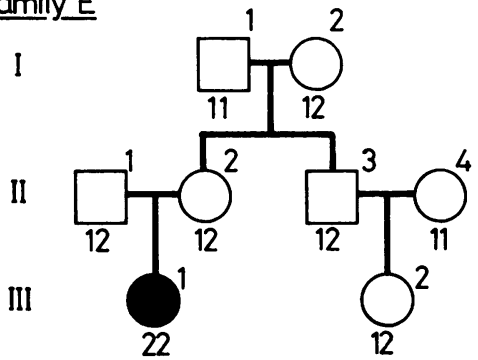

FIGURE Family pedigrees.

tion enzyme digestion, and labelled by random oligonucleotide primed synthesis with ${ }^{32}-$ PdCTP $(800 \mathrm{Ci} / \mathrm{mmol})$ to a specific activity of 5 to $10 \times 10^{8}$ dpm/ $/$ g. $^{12}$

After hybridisation, the membranes were washed at $65^{\circ} \mathrm{C}$ to a final salt concentration of $0.15 \mathrm{~mol} / \mathrm{l}$ $\mathrm{NaCl}$ in $0.2 \%$ SDS. Autoradiography with dual intensifying screens was performed at $-70^{\circ} \mathrm{C}$ for 24 hours.

The pJ3.11 probe detects a MspI polymorphic locus with $4.2 \mathrm{~kb}$ and $1.8 \mathrm{~kb}$ alleles, designated allele 1 and allele 2 respectively. Approximately $40 \%$ of the population are heterozygous for this polymorphism. Linkage studies have shown that pJ3.11 is tightly linked to the cystic fibrosis locus with a maximum lod score of 13.7 at a recombination fraction of $0(95 \%$ confidence interval, $4 \%$ previously published ${ }^{4}$ and unpublished results). This strongly suggests that there is a single locus for cystic fibrosis and genetic heterogeneity in the disease is unlikely. This is an essential precondition for using linked markers for risk analysis in genetic disease of unknown biochemical basis. Genetic risk calcu-
Family $\underline{B}$

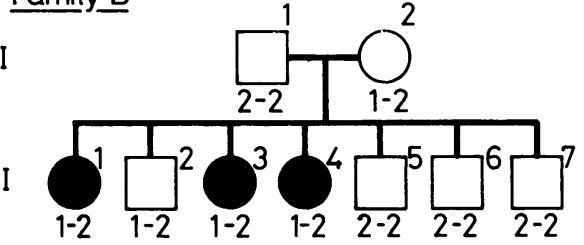

Family D

I

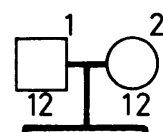

II

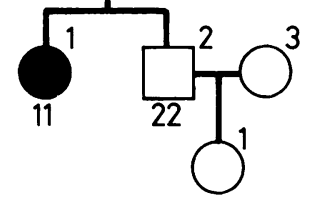

lations were done by hand and by using the computer programme LINKAGE. ${ }^{13}$

\section{Results}

CARRIER DETECTION

Detection of carriers was possible in five families with unaffected sibs out of a total of 16 families examined. In the figure, families $\mathrm{A}$ and $\mathrm{B}$ show the segregation of cystic fibrosis and the alleles for the $M s p I$ restriction fragment length polymorphism detected with the $\mathrm{pJ} 3 \cdot 11$ probe.

Family A has two affected children who provide information to determine the phase of the parents' chromosomes, who have the same genotypes: [1/CF] and [2/normal]. Both subjects II. 2 and II. 3 are unaffected by cystic fibrosis and heterozygous for the $\mathrm{pJ} 3.11$ probe, which means that each must have inherited a $[1 / \mathrm{CF}]$ chromosome from one parent. This predicts that both II. 2 and II. 3 are carriers if the small chance of recombination between cystic fibrosis and $\mathrm{pJ} 3 \cdot 11$ is disregarded.

The risk calculation has to allow for the possibility 
of recombination for both paternal or maternal chromosomes or both, together with phase data. Using the conservative estimate of a recombination fraction of $4 \%$ between the cystic fibrosis and $\mathrm{pJ} 3 \cdot 11$ loci (upper $95 \%$ confidence margin), the probability of subject II.2 being a carrier for cystic fibrosis is calculated as $\mathbf{0 . 9 5 7}$. If one makes the more likely assumption that the recombination fraction is $1 \%$, then the probability that II.2 is a carrier is 0.990 .

The figure shows the segregation of alleles at the CF and pJ3.11 loci in family B. The three affected children in this pedigree again allow the phase of the maternal chromosomes to be assumed (the maternal chromosomes are: $[1 / \mathrm{CF}]$ and $[2 /$ normal]).

Subject II. 2 must receive the $[1 / \mathrm{CF}]$ chromosome from his mother and the [2/normal] chromosome from his father which predicts that II. 2 is a carrier if recombination is disregarded. The risk calculation (assuming recombination at $4 \%$ ) reflects the chance of a crossover between the $\mathrm{CF}$ and $\mathrm{pJ} 3.11$ loci in the maternal chromosomes and gives a probability of 0.961 that II. 2 is a carrier.

Subjects II.5, II.6, and II.7 have each inherited the same maternal chromosome: [2/normal]. The paternal chromosome also carries allele 2 for the marker, and there is a $50 \%$ chance that it carries the cystic fibrosis allele. Therefore, for these three brothers, this polymorphism alone does not modify the prior risk appreciably.

\section{CARRIER EXCLUSION}

Carrier exclusion was possible in one family (C) out of the 16 families studied and is illustrated in the figure. The three affected children provide phase information for the parental chromosomes: one parent has $[1 / \mathrm{CF}]$ and $[2 /$ normal] chromosomes, the other parent has [1/normal] and [2/CF] chromosomes. Using this information the unaffected sibs must have [ $1 /$ normal $]$ and [ $2 /$ normal $]$ chromosomes if the possibility of recombination is disregarded. The risk calculation has to allow for the possibility of recombination for both the maternal and paternal chromosomes in each alternate phase assignment.

The risk of being a carrier for subject II. 4 from the RFLP inheritance is $0 \cdot 144$. A further calculation with a recombination fraction between cystic fibrosis and $\mathrm{pJ} 3 \cdot 11$ of $1 \%$ makes the probabilities for carrier status for subjects II.4 decrease to 0.04 . II.5 shares identical risks with II.4.

\section{Discussion}

The pedigrees shown above illustrate several important points for the application of currently available linked DNA probes for carrier determination in cystic fibrosis families. Only a proportion of families may be helped with a single probe with two alleles, even when the polymorphism is frequent (in this pilot study, six in 16). There are currently two probes, pJ3.11 and met, that are tightly linked to the cystic fibrosis locus, which recognise a total of five polymorphisms. Together they make approximately $80 \%$ of families informative for carrier detection. This proportion will increase as new markers are cloned around the CF locus. However, only those that are in linkage equilibrium with $\mathrm{pJ} 3 \cdot 11$ or met or both will increase the number of informative families.

Families where blood for DNA preparation may be collected from both parents and at least one affected child are suitable for carrier determination for unaffected sibs. Analysis may also be useful for second or higher degree relatives with an affected proband. Blood samples saved from proband and parents would ensure that DNA analysis would be available for unaffected sibs and second degree relatives who seek counselling. It is essential to exclude non-paternity before DNA analysis can be included in carrier determination, which may be done using a 'minisatellite' probe. ${ }^{14}$

Analysis of family B shows that not all unaffected sibs in a given sibship are necessarily helped by analysis with a single linked marker. Data from additional markers would permit haplotype analysis for the majority of families. Each parental chromosome will be different so all offspring chromosomes will in turn be identifiable. Estimation of risks for families with unresolved phase may be complex, but the identification of haplotypes will resolve phase in many families and simplify the calculations markedly. The identification of haplotype for affected chromosomes would allow analysis in families even where a parent or grandparent is missing.

Narrow confidence margins for the recombination fraction between cystic fibrosis and pJ3.11 are more important for carrier exclusion than for carrier detection, as recombination in either parental chromosome will lead to an incorrect assignment. We have used both the conservative $95 \%$ confidence limit of $4 \%$ recombination and the more likely value of $1 \%$ recombination. The margins will be reassessed as new linkage data are collected.

Risks were calculated exactly for this paper, by hand and by using the computer programme LINKAGE ${ }^{13}$ However, for the majority of counselling, simple approximations of the risks are appropriate where recombination fraction estimates are small (less than $5 \%$ ).

Most families that present for carrier determination will have one affected child and both parental mutant chromosomes will be unambiguously identifiable. If the DNA markers predict that an 
unaffected sib is a carrier then the final probability for this child being a carrier is (1-20); if the DNA markers predict a homozygote normal then the probability of being a carrier is (1-3.50). Families with two or more affected children give additional phase data; for a predicted carrier the risk is $(1-\theta)$ and for a predicted homozygote normal (1-2 $\theta)$. These estimates, to two significant figures, agree with the exact solutions.

Risk calculations for extended families, especially with consanguinity, are more complicated and computer programmes are useful. LINKAGE is available from Drs Lathrop and Lalouel, Howard Hughes Medical Institute, University of Utah, Salt Lake City, Utah 84132, USA, and is suitable for a variety of commonly available personal computers. Allowance for estimates of gene frequencies for cystic fibrosis needs consideration for these families as well.

The clinical demand for carrier detection and exclusion in families such as those described is difficult to quantify at this time. We have had several requests for carrier exclusion since our linked probes were described. Calculations may predict how useful this information will be in common counselling situations.

A typical case will be an unaffected sib with one living affected proband (family D shown in the figure). Subject II.2 has inherited one [2/normal] chromosome from each parent predicting that he will be homozygous normal. His prior risk of being a carrier is 0.66 , but this is modified by the conditional DNA data to a final probability of $0 \cdot 14$. If II. 2 has a child with II.3 (a one in 20 risk carrier from the general population), then they have a $0.14 \times 0.05 \times 0.25=0.00175$ (one in 570) chance of having a child (III.1) with cystic fibrosis. This compares with the prior chance of $0.667 \times 0.05 \times 0.25=0.008$ (one in 125). Calculations using a recombination fraction between pJ $3 \cdot 11$ and $\mathrm{CF}$ equal to $1 \%$ modify the overall risk for this couple having a cystic fibrosis child to 0.0005 (one in 2000).

A second pedigree (family E) illustrates carrier detection for cousins (figure). Subject III.2 is predicted to be a carrier as she has a similar $[2 / \mathrm{CF}]$ chromosome to her affected cousin (III.1) which were both inherited from their common grandmother (I.2). III.2 has a prior risk of being a carrier of 0.28 , which is modified by the DNA data to a final probability of $0 \cdot 85$. If III. 2 marries someone with a one in 20 risk of being a carrier, they have a $0.85 \times 0.05 \times 0.25=0.011$ (one in 94) chance of having an affected child, as compared to the prior risk $=0.28 \times 0.05 \times 0.25=0.0035$ (one in 285). Recalculation with a recombination fraction of 0.01 modifies the final risk to 0.012 (one in 83). Carrier detection for cousins is of major importance where a consanguineous marriage leads to a high prior risk for cystic fibrosis.

The tests for determining cystic fibrosis prenatally by measuring microvillar enzyme levels in amniotic fluid may be offered to those couples with a one in four risk of recurrence, ${ }^{15}$ but are not generally indicated for couples at lower risk. Hence, the major contribution that information from the DNA markers in typical counselling situations may offer is when carrier status may be excluded. In some extended pedigrees, carrier determination may give phase data helpful for couples with one affected child seeking prenatal diagnosis. In addition, DNA carrier determination in unaffected sibs and other relatives will provide additional family material to assess the various carrier detection methods based on enzymes and antigens.

The genetic relationships of $\mathrm{pJ} 3 \cdot 11$, met, and $\mathrm{CF}$ are currently being studied as a collaborative effort in several centres to attempt to order the loci. The establishment of flanking markers will enable highly accurate predictions to be made in informative families, as multiple crossovers within small genetic distances are very rare events. The eventual cloning of the gene responsible for the cystic fibrosis defect should allow the accurate diagnosis of carriers in family studies, and possibly permit detection of carriers in a population screen.

We thank our clinical colleagues and the cystic fibrosis families for their help, and the Cystic Fibrosis Research Trust and Medical Research Council for generous support. We are grateful to Professor Rodney Harris for helpful discussion and Professor Charles Coutelle (Academy of Sciences of the German Democratic Republic, Berlin Buch) for providing family material. MF is a Foulkes Foundation Fellow.

\footnotetext{
References

1 Quinton PM, Bijman J. Higher bioelectric potentials due to decreased chloride absorption in the sweat glands of patients with cystic fibrosis. $N$ Engl J Med 1983;308:1185-9.

2 Qureshi AR, Punnett HH. Carrier detection in cystic fibrosis. $J$ Pediatr 1985;106:913-7.

3 Theile H, Gressmann HW, Winiecki P. Detection of cystic fibrosis heterozygotes using a modified loading with bromide. Hum Genet 1985;69:277-80.

4 Wainwright BJ, Scambler PJ, Schmidtke J, et al. Localization of cystic fibrosis locus to human chromosome 7cen-q22. Nature 1985;318:384-5.

5 Scambler PJ, Wainwright BJ, Farrall $\mathrm{M}$, et al. Linkage of COL1A2 collagen gene to cystic fibrosis, and its clinical implications. Lancet 1985;ii:1241-2.

${ }^{6}$ White R, Woodward S, Leppert M, et al. A closely linked genetic marker for cystic fibrosis. Nature 1985;318:382-4.

7 Tsui LC, Buchwald M, Barker D, et al. Cystic fibrosis locus defined by a genetically linked polymorphic DNA marker. Science 1985;230:1054-7.
} 
' Knowlton RG, Cohen-Haguenauer O, Van Cong N, et al. A polymorphic DNA marker linked to cystic fibrosis is located on chromosome 7. Nature 1985;318:380-2.

${ }^{9}$ Kunkel LM, Smith KD, Boyer SH, et al. Analysis of human Y-chromosome specific reiterated DNA in chromosome varients. Proc Natl Acad Sci USA 1977;74:1245-9.

${ }^{10}$ Southern E. Detection of specific sequences among DNA fragments separated by gel electrophoresis. J Mol Biol 1975;98:503-17.

${ }^{11}$ Maniatis T, Fritsch EF, Sambrook J. Molecular cloning. A laboratory manual. New York: Cold Stream Harbor Laboratory, 1982:387.

${ }^{12}$ Feinberg AP, Vogelstein B. A technique for radiolabelling
DNA fragments to high specific activity. Anal Biochem 1984;137:266-7.

${ }^{13}$ Lathrop GM, Lalouel JM. Easy calculations of lod scores and genetic risks on small computers. Am J Hum Genet 1984;36:460-5

14 Jeffreys AJ, Wilson V, Thein SL. Hypervariable 'minisatellite' regions in human DNA. Nature 1985;314:67-73.

15 Several authors. Special section on the prenatal diagnosis of cystic fibrosis. Prenat Diag 1985;5:93-135.

Correspondence and requests for reprints to Professor R Williamson, Department of Biochemistry, St Mary's Hospital Medical School, London W2 1PG. 